\title{
Transdiagnostic Alterations in Neural Emotion Regulation Circuits - Neural Substrates of Cognitive Reappraisal in Patients with Depression and Post- Traumatic Stress Disorder
}

\author{
Micha Keller \\ Universitätsklinikum Aachen \\ Raul Mendoza-Quiñones \\ Cuban Neuroscience Center \\ Amaray Cabrera-Muñoz \\ Cuban Neuroscience Center \\ Jorge Fuster \\ Cuban Neuroscience Center \\ Anette Valdés Virués \\ Cuban Neuroscience Center \\ Mikhail Zvyagintsev \\ Universitätsklinikum Aachen
}

James Christopher Edgar

Children's Hospital of Philadelphia

Jana Zweerings

Universitätsklinikum Aachen

Klaus Mathiak ( $\nabla$ kmathiak@ukaachen.de)

Universitätsklinikum Aachen

\section{Research Article}

Keywords: depression, post-traumatic stress disorder, emotion regulation, cognitive reappraisal, lateral PFC

Posted Date: September 15th, 2021

DOl: https://doi.org/10.21203/rs.3.rs-845857/v1

License: (c) (1) This work is licensed under a Creative Commons Attribution 4.0 International License. Read Full License 
Version of Record: A version of this preprint was published at BMC Psychiatry on March 8th, 2022. See the published version at https://doi.org/10.1186/s12888-022-03780-y. 


\section{Abstract}

Background: Impaired cognitive reappraisal, associated with the social functioning and well-being of patients affected by mood or anxiety disorders, is characterized by distinct neural activation patterns across clinical populations. To date, studies dedicated to identifying common and distinct neural activation profiles need to be clarified. The aim of the present study was to investigate transdiagnostic differences and commonalities in brain activation patterns during reappraisal-mediated downregulation of emotions

Methods: Cognitive reappraisal of negative images was contrasted with maintaining emotions during a control viewing condition. Brain activation in 35 patients with major depressive disorder (MDD), 20 patients with post-traumatic stress disorder (PTSD), and 34 healthy controls (HC) during cognitive reappraisal was compared. Moreover, the neural circuitry of emotion regulation in these clinical populations was examined using task-based and intrinsic functional connectivity analyses.

Results: Whole-brain fMRI analyses showed less right-lateralized activation of the inferior, middle and superior frontal gyrus during cognitive reappraisal compared to viewing of negative images in MDD and PTSD patients compared to HCs. Right IFG activation was negatively correlated with the severity of anxiety and depressive symptomatology. In addition, increased seed-based connectivity of right IFG as well as increased intrinsic connectivity was observed in PTSD patients compared to HCs and MDD patients.

Conclusions: FMRI results therefore suggested a common deficit of depression and anxiety symptomatology reflected by reduced activation in right IFG during cognitive reappraisal as well as diagnosis specific effects in patients with PTSD based on seed-based and intrinsic connectivity showing an overactive and hyperconnected salience network. Findings highlight the role of transdiagnostic research to identify disorder specific brain patterns as well as patterns common across disorders.

\section{Background}

The identification of neural mechanisms related to key clinical features in psychiatric disorders may help to tailor individualized brain-based training programs to treat cognitive deficits. For instance, the investigation of the neural underpinnings of emotion dysregulation and sustained negative affect as core features of anxiety and mood disorders may help identify neural targets for novel treatments such as real-time fMRI neurofeedback or transcranial direct current stimulation (Marques et al., 2018; Mathiak \& Keller, 2021; Weiskopf et al., 2004). Current studies on emotion regulation in psychiatric disorders such as major depressive disorder (MDD) and post-traumatic stress disorder (PTSD) often investigate the use of cognitive reappraisal, an antecedent focused emotion regulation strategy, that entails the attempt to reinterpret an emotion-eliciting situation in order to change its emotional impact (Kanske et al., 2012; Keller et al., 2021; Sarkheil et al., 2015; Wang et al., 2014; Zilverstand et al., 2017a; Zweerings et al., 2020).

\section{Neural substrates of cognitive reappraisal}


Investigations of the neural bases of emotion regulation are critical to understanding the relation between a reduced capacity to use cognitive reappraisal and the severity of depressive symptoms (Berking et al., 2014). Cognitive reappraisal involves the down-regulation of negative emotions and is associated with enhanced activation of medial and lateral prefrontal regions, accompanied by reduced activation of emotion arousal-related brain structures including the amygdala and the insula (Hofmann, 2014; K N Ochsner \& Gross, 2005). Several meta-analyses on cognitive reappraisal in healthy and clinical populations have been published (Buhle et al., 2014; Kohn et al., 2014; Picó-Pérez et al., 2017; Zilverstand et al., 2017b). Findings in healthy controls (HCs) indicate consistent involvement of cognitive control regions, including dorsomedial prefrontal cortex (dmPFC), dorsolateral prefrontal cortex (dIPFC), ventrolateral prefrontal cortex (VIPFC), and posterior parietal lobe during cognitive reappraisal compared to a control condition, as well as reduced activity of the left and right amygdala (Buhle et al., 2014). Kohn and colleagues (2014) proposed a neural model of conscious, cognitive emotion regulation. In this model, whereas the dIPFC is related to regulation of cognitive processes such as attention, the vIPFC may not reflect the regulatory process per se, but signals salience and therefore the need to regulate. Furthermore, additional frontal areas are consistently involved in emotion regulation tasks: the anterior middle cingulate cortex (aMCC), superior temporal gyrus (STG), angular gyrus and (pre-) supplementary motor area (SMA). Furthermore, the authors proposed that the STG, angular gyrus and (pre-)SMA are involved in the execution of regulation initiated by frontal areas.

\section{Cognitive reappraisal in clinical populations}

Zilverstand and colleagues (2017) extended the literature on neural substrates underlying cognitive reappraisal to clinical populations. The authors showed that across clinical populations, individuals consistently demonstrated reduced recruitment of vIPFC and dIPFC regions, key nodes of the top-down regulatory cognitive control network in HCs. In addition, individuals with mood disorders (including MDD) showed enhanced amygdala activation during downregulation of emotion, suggesting hyperactive bottom-up responses, or reduced modulatory capacity of regulatory networks during cognitive reappraisal. In contrast, patients with anxiety disorders (including PTSD) showed reduced involvement of parietal regions, indicating impaired functionality of fronto-parietal attention networks, alongside reduced responses in the dorsal anterior cingulate cortex (dACC). Responsivity of the ACC in patients with PTSD has also been associated with symptom severity (Dickie et al., 2013; Zweerings et al., 2018).

In a similar line of investigation, Picó-Pérez and colleagues (2017) studied the neural correlates of emotion regulation in a large sample of patients with mood or anxiety disorders and healthy individuals. The patients showed decreased activation of the prefronto-parietal network (PCC, the dmPFC, the angular gyri and the left vIPFC) in combination with increased activation in regions associated with the experience of emotions (i.e., insula, cerebellum, precentral and inferior occipital gyri) as well as in regions where activation may be the consequence of compensatory mechanisms (i.e., supramarginal gyri and superior parietal lobule). Other studies have supported these findings by demonstrating structural and functional abnormalities in the prefrontal cortex circuits related to top-down inhibitory control in MDD and PTSD (Etkin \& Wager, 2007; Phillips et al., 2008; Rive et al., 2013). 
In addition, the examination of the neural bases of volitional affect regulation in combat-related PTSD revealed that veterans with PTSD showed less recruitment of the dIPFC during cognitive regulation of affect, compared to veterans exposed to similar levels of combat stress without PTSD, suggesting altered neural activation during volitional self-regulation of negative affective states (Rabinak et al., 2014).

\section{Functional connectivity across clinical populations}

Emotion dysregulation observed in MDD patients can also be considered a result of disruption of interconnected circuits. Dysfunctional connectivity of the affective salience, cognitive control, and default mode networks appears to underlie characteristic symptoms of depression, including depressed mood, anhedonia, self-rumination, and impaired concentration (Fischer et al., 2016; Zweerings et al., 2019). The most frequently reported resting-state abnormalities in brain networks in patients with depression include amygdala hyperconnectivity within the salience network (Price \& Drevets, 2010), hypoconnectivity of the frontoparietal network (Kaiser et al., 2015), and hyperconnectivity of the default mode network (Raichle, 2015).

Altered emotion-related neurocircuitry has also been shown in individuals with PTSD. Sripada and colleagues (2012) investigated patterns of resting-state functional connectivity (FC) of the amygdala in whole brain analyses and found that compared to HCs the veterans with PTSD showed greater positive connectivity between the amygdala and the insula, as well as between the amygdala and the hippocampus, and reduced negative connectivity between the amygdala and dorsal and rostral ACC (Sripada et al., 2012). The authors suggested that these abnormalities in emotion generation and regulation circuits may contribute to the pathophysiology of PTSD including deficits of emotion processing and emotion regulation.

PTSD and depression are highly comorbid disorders, with approximately half of patients with current PTSD also showing co-occurring depression (Rytwinski et al., 2013). Furthermore, both disorders are characterized by heightened levels of anxiety and depression symptoms (Stanley et al., 2017). Metaanalyses suggest shared as well as distinct neural alterations that underpin emotion regulation deficits in mood and anxiety disorders (including MDD and PTSD) (Cristea et al., 2015). However, to our knowledge, no study has directly compared these patient cohorts with respect to commonalities and differences in neural processes, including FC of emotion dysregulation in PTSD and MDD. A transdiagnostic approach targeting both PTSD and MDD should provide insight into emotion dysregulation across target groups.

The aim of the present study was to investigate the $\mathrm{fMRI}$ co-activation patterns during reappraisalmediated downregulation of emotion in MDD and PTSD patients compared to healthy individuals. Moreover, the neural circuitry of emotion regulation in these clinical populations using task-based FC was explored. We expected to replicate common alterations in frontal regulatory control regions in both patient groups as well as observe disorder-specific alterations in limbic and parietal regions and also dorsal anterior cingulum. In addition, we sought to explore associations between anxiety and depression related symptom severity across patient groups as well as associations with specific types of emotion regulation strategies (i.e., suppression and cognitive reappraisal). 


\section{Methods}

\section{Subjects}

Thirty-five patients with MDD (age $37.3 \pm 13.5$ ) and 20 patients with PTSD (age $43.4 \pm 13.0$ ) as well as 34 healthy individuals (age $39.4 \pm 12.1$ ) without history of neurological or psychiatric disorders participated in this study. All participants were right-handed, had adequate knowledge of the German language, and normal or corrected to normal vision. Contraindications for MRI, pregnancy, as well as acute suicidality served as exclusion criteria for all participants. Diagnoses were based on the Diagnostic and Statistical Manual of Mental Disorders (DSM-IV-TR) criteria (Association American Psychiatric, 2000) and were confirmed by an experienced psychologist using the German version of the Structured Clinical Interview for assessment of DSM-IV-TR criteria (SCID-I; American Psychiatric Association, 2000). Patients were recruited through the Department of Psychiatry, Psychotherapy and Psychosomatics of the University Hospital Aachen. Additionally, patients with PTSD were recruited from a specialized outpatient clinic, the 'Euregio Institut für Psychosomatik und Psychotraumatologie'. Patients with PTSD were excluded in case of severe affective disorders or substance dependence, multiple traumatic events since childhood, or acute somatic or neurologic disorders. All patients with PTSD developed symptoms after the experience of a single traumatic event. Patients with depression had stable medication for at least 1 week before participation. The data were acquired during the baseline measurements of two randomized control trials (Keller et al., 2021; Zweerings et al., 2020). All experiments were performed with the written informed consent of each participant and approval by the local Ethics Committee of the RWTH Aachen University Hospital, in line with the Code of Ethics of the World Medical Association (Declaration of Helsinki).

\section{Questionnaire acquisition and analysis}

The hospital anxiety and depression scale (HADS, Zigmond and Snaith, 1983) as well as the German version of the emotion regulation questionnaire (ERQ, Gross and John, 2003) assessed symptoms of anxiety and depression as well as emotion regulation ability. The ERQ measures respondents' tendency to use cognitive reappraisal or expressive suppression strategies to regulate positive and negative emotions. The HADS is a self-report rating scale designed to measure anxiety and depression. Demographic and behavioral data were analyzed using a one-way ANOVA (e.g., age, ERQ, HADS) or chi-square test (e.g., gender) using STATISTICA Version 10 (Statsoft, 2011)

\section{Stimuli and task}

Stimuli were projected onto a screen located at the back of the MRI scanner, with the stimuli viewed through an angled mirror fixed to the MRI head-coil. Stimuli were presented using the Cogent Matlab toolbox (http://www.vislab.ucl.ac.uk/cogent.php). Participants were presented 19 pictures from the International Affective Picture System (IAPS) with negative valence (Lang et al., 2008).

All participants received comprehensive instructions on cognitive reappraisal strategies alongside a supervised training to apply the learned strategies to the re-evaluation of negative visual scenes prior to entering the scanner. In the scanner, each participant completed one emotion regulation run with 9 
reappraise-view cycles ( 7 minutes). In the 'view' condition, subjects were instructed to maintain the negative emotion elicited by the image. In the 'reappraise' condition, subjects were instructed to reappraise the content of the negative emotion by changing one's interpretation of the negative stimulus such as imagining that (1) the situation is not as bad as it looks or (2) will get better in the future or by (3) imagining that the situation is not real or (4) taking the perspective of a professional.

Preceding and following the view-reappraise cycles, participants rated their current valence and arousal level on a scale from 1 to 7 (see Fig. 1 for experimental design).

\section{fMRI data acquisition}

The MRI scanning was performed using a 3.0 Tesla Siemens MAGNETOM Prisma scanner (Siemens Medical Systems, Erlangen, Germany) equipped with a 20-channel head coil. T2*-weighted images were obtained using echo-planar imaging with the following parameters: repetition time $(T R)=2,000 \mathrm{~ms}$, echo time $(T E)=36 \mathrm{~ms}$, flip angle $=77$ and matrix size $64 \times 64$. Images were acquired with 26 transverse slices in ascending interleaved order (voxel size $3 \times 3 \times 4 \mathrm{~mm}^{3}$; gap $=0.5 \mathrm{~mm}$; field of view $=192 \times 192 \mathrm{~mm}^{2}$ ). The first five volumes were discarded to account for T1-saturation effects. All participants were instructed to minimize movement inside the scanner and to keep eyes open and focus on the fixation cross.

\section{fMRI Data analysis}

Preprocessing of imaging data and statistical analysis were carried out using SPM12 software (Wellcome Trust Center for Neuroimaging, London, UK) implemented in Matlab R2017b. The functional scans were first submitted to artifact correction using the ArtRepair toolbox

(http://cibsr.stanford.edu/tools/ArtRepair/ArtRepair.htm), thus repairing motion/signal intensity outliers and other artifacts (including interpolation using nearest neighbors for bad scans). Slice-timing correction, head motion correction (including extraction of motion parameters), and unwarping procedures were also applied. Anatomical and functional data coregistration and spatial normalization into MNI template space were done to enable group analyses. A $128 \mathrm{~s}$ high-pass filter removed lowfrequency drifts. Spatial smoothing was performed using an $8 \mathrm{~mm}$ full-width at half maximum Gaussian kernel. Head motion parameters and their temporal derivatives were included in the linear model to reduce motion artifacts.

\section{General Linear Model for the cognitive reappraisal task}

For exploratory whole-brain analysis, the task-related BOLD signal changes at the subject level were estimated using a whole-brain first-level analysis based on General Linear Models (GLM) in SPM12. The first-level contrasts used for group analysis were: [reappraise > view] to detect brain networks with increased activation during cognitive reappraisal compared to baseline (view), and [reappraise < view] to identify reduced activation patterns during cognitive reappraisal compared to baseline (view).

Subsequently, the contrast images of each subject were entered into a second-level model. We performed a random-effect group analysis on the contrast images using one-sample $t$-tests ( $t$-tests vs 0 ) separately for HCs, MDD and PTSD to identify networks involved in emotion regulation for each group. T-maps for 
contrasts of interest [reappraise > view] represented the activation of regions involved in the cognitive control of emotion, and the opposite contrast [reappraise < view] represented regions with lower activation for regulation compared to view during this task. Second-level results were evaluated with $p_{\mathrm{FWE}}<.05$ at voxel level for the individual groups and at $p<.001$ uncorrected voxel level and with $p<.05$ family-wise error (FWE) correction for the group difference ( $\mathrm{HC}>$ Patients). Clusters were labelled using the automated anatomical labeling atlas 3 (AAL3; Rolls et al., 2020). To examine whether group differences in activation were associated with differences in symptom severity of depression and anxiety (HADS) as well as differences in emotion regulation (ERQ), Pearson correlations were computed between the observed cluster in the right lateral PFC and questionnaire data. Correlation analyses were corrected for multiple comparisons ( 4 tests: $a=0.05 / 4=0.0125$ ).

\section{ROI analysis}

We focused our analysis on a priori regions previously implicated in the cognitive reappraisal literature (Kohn et al., 2014; Pico-Pérez et al., 2017). The MarsBaR toolbox (version 0.44; Brett et al., 2002) was used to create ROI spheres of $10 \mathrm{~mm}$ radius centered on the peak coordinates provided in previous metaanalyses (Kohn et al., 2014; Pico-Pérez et al., 2017). The selected ROls were: 1) the posterior cingulate cortex (PCC), extending into the precuneus $(-4,-38,2) ; 2)$ the bilateral dorsomedial prefrontal cortex (dmPFC) [left_dmPFC: $(-6,32,48)$ and right_dmPFC $(16,20,50)]$; 3$)$ the bilateral angular gyrus (AG) [left_AG: $(-42,-72,34)$ and right_AG $(60,-54,40)]$; 4$)$ the left ventrolateral prefrontal cortex (vIPFC) [left_vIPFC; $-54,36,-2)]$; 5) bilateral inferior frontal gyrus (IFG) [left_IFG: $(-42,22,-6)$ and right_IFG $(50,30,-8)] ; 6)$ left middle temporal cortex (MTC; 38, 22, 44); 7) bilateral precentral gyrus (PG) [left_PG: $(-44,10,46)$ and right_PG (42 - 1634$)$ ] and 8) (pre-)supplementary motor area (SMA) $(-2,14,58)$. Finally, the mean ROI beta values were extracted from the contrast maps of all subjects using MarsBaR. Beta values were compared between HCs, MDD and PTSD patients using a one-way ANOVA $(p<.05)$ followed by post-hoc Tukey's Honest Significant Difference (HSD) to correct for multiple comparisons.

Connectivity analyses. To investigate FC group differences, we conducted a task based as well as intrinsic connectivity (IC) analysis using the FC toolbox CONN (www.nitrc.org/projects/conn, RRID:SCR_009550). The data were preprocessed using the default preprocessing pipeline for volumebased analysis which includes realignment and unwarping of functional data, slice- timing correction, outlier identification, direct segmentation and normalization as well as functional smoothing with a Gaussian kernel of $8 \mathrm{~mm}$ full width half maximum (FWHM).

Task-based connectivity analysis. To investigate task-based connectivity, a generalized psychophysiological interaction (gPPI) was computed. Signal variance that correlated with the seed region during the regulation compared to view condition ('reappraise - view') was investigated. The right inferior frontal gyrus (pars triangularis and opercularis) was chosen as a seed and was created based on the target regions. Second-level results were evaluated at $p<.005$ uncorrected voxel level and with $p<.05$ FWE-correction at the cluster level. 
Intrinsic connectivity. Within network changes during cognitive reappraisal (contrast between reappraise vs. view) as well as group differences of IC were investigated. IC is a measure of network centrality at each voxel and characterizes the strength of the connectivity pattern between each voxel and the rest of the brain (root mean square of the correlation coefficient values) (Martuzzi et al., 2011). Second-level results were evaluated at $p<.005$ uncorrected voxel level and with $p<.05$ FWE-correction at the cluster level.

\section{Results}

\section{Demographics and self-report data}

Analyses of demographic data indicated no differences in age between the three groups $(F(2,86)=1.408$, $p=.25)$. Furthermore, the three groups did not differ with regard to gender $\left(X^{2}(2, N=89)=.0173, p=.99\right)$. One-way ANOVAs investigated the main effect of group on emotion regulation (ERQ) and symptom severity of depression and anxiety (HADS) (see Table 1 and Fig. 2). There was a significant main effect of group for cognitive reappraisal (ERQ-CR; $F(2,80)=6.6, p=.002)$. Post-hoc comparisons with the Tukey HSD test revealed that patients with depression reported less use of cognitive reappraisal than the control group ( $p=.002$ ). The test revealed no group differences in the following comparisons: i) Controls vs $\operatorname{PTSD}(p=.15)$ and ii) MDD vs PSTD $(p=.58)$. A separate ANOVA revealed no main effect of group for suppression strategies (ERQ-SUP; $F(2,86)=2.079, p=.13$ ).

Table 1

Demographic and clinical characteristics

\begin{tabular}{|c|c|c|c|c|c|c|c|c|c|}
\hline & \multicolumn{2}{|c|}{$\begin{array}{l}\text { Controls }(n= \\
34)\end{array}$} & \multicolumn{2}{|c|}{ MDD $(n=35)$} & \multicolumn{2}{|c|}{ PTSD $(n=20)$} & \multirow[b]{2}{*}{$x^{2}$} & & \multirow[b]{2}{*}{$p$} \\
\hline & $n$ & $\%$ & $n$ & $\%$ & $n$ & $\%$ & & & \\
\hline \multirow[t]{3}{*}{ Gender (male) } & 17 & 50 & 18 & 51.4 & 10 & 50 & .017 & & .99 \\
\hline & \multicolumn{2}{|c|}{ Controls } & \multicolumn{2}{|l|}{ MDD } & \multicolumn{2}{|l|}{ PTSD } & & & \\
\hline & $M$ & $S D$ & $M$ & $S D$ & $M$ & $S D$ & $F$ & $d f$ & $p$ \\
\hline Age & 39.38 & 12.1 & 37.34 & 13.5 & 43.40 & 13.0 & 1.41 & 2,86 & .25 \\
\hline ERQ_CR & 4.87 & 0.81 & 3.84 & 1.31 & 4.51 & 1.47 & 6.6 & 2,80 & .002 \\
\hline ERQ_SUP & 3.77 & 0.93 & 4.36 & 1.34 & 3.94 & 1.4 & 2.08 & 2,80 & .13 \\
\hline HADS_A & 3.03 & 2.59 & 11.76 & 3.86 & 10 & 4.07 & 56.2 & 2,83 & .001 \\
\hline HADS_D & 2.03 & 2.34 & 10.91 & 4.25 & 7.4 & 4.42 & 49.02 & 2,84 & .001 \\
\hline
\end{tabular}


Furthermore, there were main effects of group for both subscales of the HADS (HADS-Anxiety $(F(2,83)=$ $56.2, p<.001)$; HADS-Depression $(F(2,84)=49.06, p<.001))$. Healthy individuals had lower scores on the anxiety subscale than patients with depression $(p<.001)$ and PTSD $(p<.001)$. Both clinical groups showed similar levels of anxiety $(p=.18)$. Healthy controls also obtained lower scores on the depression subscale than MDD $(p<.001)$ and PTSD $(p<.001)$ patients. However, patients with depression showed higher levels of depressive symptoms than patients with PTSD $(p<.01)$.

\section{Whole-brain fMRI analysis}

To examine group effects of reappraising compared to viewing negative stimuli, two contrasts were examined with one sample $t$-tests for each individual group on the second level. First, the contrast 'reappraise > view' represented the activation of regions involved in the cognitive control of emotion in the group of HCs. Second, the opposite contrast 'reappraise < view' represented brain regions that were less active during reappraisal compared to 'view' (see Fig. 3 and Appendix 1 for overview of results). These analyses revealed increased activation during cognitive reappraisal for HCs in the bilateral IFG (pars triangularis), bilateral middle frontal gyrus, left precentral gyrus, bilateral SMA, bilateral temporal gyrus, right caudate, and right thalamus. Lower activations during cognitive reappraisal were found in the right insula. The same analysis in patients with MDD revealed only activation in the left IFG (pars triangularis), left middle frontal gyrus, right cerebellum, left medial segment of the superior frontal gyrus and left insula during cognitive reappraisal. However, patients with MDD showed significantly lower activation for reappraisal compared to 'view' in the right middle cingulate gyrus, right rolandic operculum, bilateral insula, left Heschl's gyrus and right supramarginal gyrus (see Fig. 3). In the PTSD group cognitive reappraisal was related to a significant cluster in the left IFG (pars orbitalis and triangularis).

Furthermore, the same model was used to evaluate group differences between HCs and patients with MDD and PTSD. HCs had greater activation compared to patients (MDD + PTSD) in a cluster covering (according to AAL3) the right triangular (46.4\%) and opercular (23.5\%) parts of the IFG as well as the right precentral gyrus (17.4\%) [(56 20 8), $T_{\text {peak }}=4.96$ ] (see Fig. 4). $T$-tests between HCs and MDD revealed a similar cluster in the right precentral gyrus (30.5\%), the right opercular (29.3\%) and triangular (26.4\%) part of the IFG [(56 20 8), $\left.\left.T_{\text {peak }}=4.7\right)\right]$. At the selected threshold there were no significant differences between HCs and patients with PTSD [(52 264$\left.), T_{\text {peak }}=4.3, p_{\mathrm{FWE}}=.08\right]$ as well as between patients with MDD and PTSD. Furthermore, the three groups did not differ regarding lower activation during cognitive reappraisal compared to viewing.

To examine the relation of dysfunctional neural patterns during cognitive reappraisal and behavioral parameters, the cluster comprising the group difference ( $\mathrm{HC}>$ Patients) was extracted and Pearson correlations between ROI mean beta values and ERQ as well as HADS results were computed.

Significance was assessed based on a corrected threshold for the number of comparisons $(n=4)$ of a $=.0125$. Significant negative correlations of HADS anxiety and depression scales with beta values (HADSAnxiety $(r=-.38, N=81, p<.001)$ and HADS-Depression $(r=-.34, N=81, p<.01))$ suggested that a higher 
clinical severity of anxiety or depression was related to less recruitment of the right IFG during cognitive reappraisal. A similar analysis revealed no significant association between mean beta values of the right lateral PFC ROI and the self-report of cognitive reappraisal (ERQ-CR; $r=.12, N=81, p=.27$ ) or suppression (ERQ-supression; $r=-.07, N=81, p=.52$ ).

\section{ROI Analysis}

For the ROI analysis, a priori regions previously been associated with cognitive reappraisal were selected (Kohn et al., 2014; Pico-Perez et al., 2016): bilateral angular gyrus, bilateral IFG, bilateral dorso-medial prefrontal cortex, bilateral precentral gyrus, the left middle temporal cortex (MTC), the left ventrolateral prefrontal cortex, and the bilateral supplementary motor area/pre-supplementary motor area. A 3 (Group [MDD, PTSD, HC]) x 12 (ROI) ANOVA was computed to investigate differences in mean ROI values for the contrast 'reappraise > view'. The ANOVA revealed a significant main effect of group for the left middle temporal cortex, right dorsomedial prefrontal cortex, right inferior frontal gyrus and left pre-central gyrus (Fig. 5).

Post-hoc tests (HSD) showed the following results: i) Compared to patients with MDD, HCs showed higher activation in the left MTC, bilateral dmPFC, right IFG, left PCG, SMA; ii) PTSD showed higher activation in the right dmPFC and right IFG compared to HCs.

\section{Connectivity analysis}

To evaluate task-related differences in FC between HCs and patients with MDD and PTSD, we conducted a task-based connectivity analysis using the right IFG as a priori region of interest. To further investigate the functional organization of the brain during the cognitive reappraisal task without a priori assumptions, we conducted an IC analysis.

Task-based connectivity analysis. Using the right IFG (pars triangularis and opercularis) as seed, we investigated the effect of cognitive reappraisal compared to viewing of negative pictures on brain system interactions. For the between-conditions contrast (reappraise $>$ view), separate $t$-tests were computed for HCs as well as MDD and PTSD patients. HCs exhibited increased FC between the rIFG seed and the left cerebellum, left occipital fusiform gyrus, left lingual gyrus and precuneus (cluster: $-26-48-26$, size $=808$, $p \mathrm{FWE}<.0001$; cluster: $-22-96+08$, size $=334, p \mathrm{FWE}=.004$ and cluster: $-16-80+20$, size $=311, p \mathrm{FWE}$ $=.007$ ) as well as the right pre/postcentral gyrus and the right superior parietal lobule (cluster: $+16-30+$ 44 , size $=309, p F W E=.007)$. In addition, increased $\mathrm{FC}$ was found in an anterior network cluster comprised of the right superior frontal gyrus and the middle frontal gyrus (cluster: $+24-18+60$, size $=245, p F W E$ $=.028$ ). Patients with MDD demonstrated increased FC between the rIFG and the bilateral cerebellum as well as the left occipital fusiform gyrus (cluster: $-02-72-34$, size $=908, p F W E<.0001$ ). Reduced FC emerged between the rIFG and two clusters comprising the left middle frontal gyrus (cluster: $-32+12+42$, size $=544, p F W E<.0001)$, the precuneus, left lateral occipital cortex, left middle frontal gyrus and posterior cingulate gyrus (cluster: $-32+12+42$, size $=544$, $p F E W<.0001$ ). PTSD patients showed increased FC between the rIFG seed and a antero-central network comprised of the bilateral paracingulate 
gyrus, the anterior cingulate gyrus, and bilateral medial and superior frontal gyrus (cluster: $+08+46-04$, size $=268, p \mathrm{FWE}=.0018$; cluster: $+00+48+22$, size $=238, p \mathrm{FWE}=.03$ ).

Table 2 contains between-group connectivity estimates. Compared to HCs, the PTSD group showed increased FC of the rIFG with the left frontal orbital cortex, left temporal pole, left inferior frontal gyrus (pars opercularis), left frontal operculum, and left insular cortex but less coupling between rIFG and the right lateral occipital cortex. PTSD patients showed greater functional coupling of the rIFG and the precuneus, the anterior and posterior cingulate gyrus, the bilateral paracingulate gyrus, the bilateral superior frontal gyrus, the left frontal orbital cortex, the left frontal gyrus, the left temporal pole, the left superior frontal gyrus and left insula compared to MDD patients. The HC > MDD comparison showed no significant differences in connectivity during cognitive reappraisal.

Table 2

Task-based connectivity for right IFG (pars triangularis + opercularis) seed during cognitive reappraisal (reappraise $>$ view). Threshold was $p<.005$ voxel-level and FWE-cluster correction $p<.05$.

\begin{tabular}{|c|c|c|c|c|c|}
\hline \multirow{2}{*}{$\begin{array}{l}\text { PTSD }>\mathrm{HC} \\
\text { Brain regions }\end{array}$} & \multicolumn{3}{|c|}{ peak MNI coordinates [mm] } & \multirow[t]{2}{*}{ Extent [voxel] } & \multirow[t]{2}{*}{ peak t-values } \\
\hline & $\mathbf{x}$ & $\mathbf{y}$ & $\mathbf{z}$ & & \\
\hline Frontal orbital cortex (I) & -42 & 24 & -12 & 529 & -5.49 \\
\hline \multicolumn{6}{|l|}{ Temporal pole (I) } \\
\hline \multicolumn{6}{|l|}{ Inferior frontal gyrus (I) } \\
\hline \multicolumn{6}{|l|}{ Frontal operculum (I) } \\
\hline \multicolumn{6}{|l|}{ Insular cortex (I) } \\
\hline \multicolumn{6}{|l|}{ PTSD > MDD } \\
\hline Precuneus & -06 & -38 & 42 & 1338 & 6.53 \\
\hline \multicolumn{6}{|l|}{ Posterior cingulate gyrus } \\
\hline Paracingulate gyrus (b) & 00 & 48 & 18 & 634 & 4.23 \\
\hline \multicolumn{6}{|l|}{ anterior cingulate gyrus } \\
\hline \multicolumn{6}{|l|}{ Superior frontal gyrus (b) } \\
\hline Frontal orbital cortex (I) & -46 & 24 & -16 & 414 & 5.32 \\
\hline \multicolumn{6}{|l|}{ Temporal pole (I) } \\
\hline $\begin{array}{l}\mathrm{HC}>\mathrm{MDD} \\
\text { none of the voxels surviv }\end{array}$ & corre & & & & \\
\hline
\end{tabular}


Intrinsic connectivity. As a data driven measure of functional network organization during the emotion regulation task, we investigated IC. HCs showed a global reduction of IC in a widespread network during cognitive reappraisal. The largest effect of IC was present in a right-lateralized anterior network, which included the middle frontal gyrus, the paracingulate gyrus, the superior frontal gyrus, and the anterior cingulate gyrus. Patients with MDD showed reduced IC in an anterior network including the supplementary motor cortex, the left paracingulate gyrus and bilateral superior frontal gyrus. Finally, the analysis showed no significant IC patterns in PTSD patients during cognitive reappraisal (see Fig. 6).

Differences between groups were identified using paired $t$-tests $(p<.005$ unc. at voxel level and $p<.05$ FWE-corrected at cluster level; 124-voxel cluster threshold; Appendix 2). Compared to HCs, PTSD patients exhibited significantly higher IC values in a posterior network including the precuneus, posterior cingulate gyrus, bilateral lingual gyrus, bilateral intracalcarine cortex, bilateral cuneal cortex, right supracalcarine cortex and the bilateral occipital cortex. The MDD group also showed a significantly higher IC compared to HCs in two clusters in the precentral gyrus. The comparison between clinical groups showed that PTSD patients exhibited increased IC relative to patients with MDD in the right lateral occipital cortex, right angular gyrus, right middle temporal gyrus, right supramarginal gyrus, and right superior temporal gyrus.

\section{Discussion}

The identification of pathophysiological mechanisms common to psychiatric disorders as well as mechanisms unique to specific psychiatric disorders are critical for elucidating the biological mechanisms underlying psychiatric symptoms and developing targeted treatment protocols (Insel et al., 2010; Nusslock et al., 2015). The purpose of this study was to identify differences in brain activation patterns between patients with psychiatric disorders and HCs during cognitive regulation of negative emotions. More specifically, we focused on the comparison of patients with MDD and PTSD. In this study, we employed a classical emotion regulation paradigm to identify emotion regulation networks common and specific to HCs as well as patients with MDD and PTSD by contrasting reappraisal of negative pictures with natural viewing of negative pictures. Both clinical groups showed higher scores of anxiety and depression compared to HCs, and with a higher self-reported severity of depression in MDD compared to PTSD patients. On the neurobiological level, exploratory analyses showed that MDD and PTSD patients had less right-lateralized activation of the inferior, middle and superior frontal gyrus during cognitive reappraisal (reappraise vs view) compared to HCs. Importantly, this cluster in the right IFG was negatively correlated with the scores within the hospital anxiety and depression scale (HADS), suggesting that less recruitment of right IFG may be associated with greater severity of anxiety as well as depressive symptomatology. Furthermore, our task-based and IC analyses confirmed group differences in connectivity during cognitive reappraisal. To our knowledge this is the first study directly comparing focal neural activation and FC patterns in HCs, MDD and PTSD patients. 
Comparison with the existent literature on neural correlates of cognitive reappraisal in clinical populations.

Our whole brain $\mathrm{fMRI}$ analysis revealed significant activation of the bilateral inferior frontal gyrus (pars triangularis), bilateral temporal, middle frontal, and superior frontal gyrus during cognitive reappraisal. Previous studies consistently report that cognitive reappraisal engages a prefronto-parietal network which exerts top-down control on limbic areas (Costafreda et al., 2008; Kohn et al., 2014; Picó-Pérez et al., 2017). Accordingly, our results are largely consistent with the literature.

In terms of task-based fMRI connectivity, HCs exhibited increased FC between the rIFG seed and clusters distributed along posterior and frontal regions. Consistent with previous studies, our findings suggested the existence of several large-scale networks that are co-activated during cognitive reappraisal. Morawetz and colleagues (2020) identified four clusters of co-activation patterns during emotion-generative and emotion-regulatory processes to brain-networks underlying prominent psychological functions and evaluated their specificity in relation to emotion regulation. Increased connectivity between rIFG and superior and middle frontal gyri as well as with the precuneus and the left lingual gyrus overlaps with a network which plays an intermediary role in reappraisal by integrating information from prefrontal and subcortical areas to generate and regulate emotional responses.

Patients with depression and PTSD displayed reduced activation of the vIPFC (a crucial node of the emotion regulatory network) during downregulation of negative emotion (Zilverstand et al., 2017b). Wang et al., (2014) examined the neural mechanisms of self-related reappraisal in Chinese MDD outpatients and found that depressed individuals exhibited diminished activation in left IFG when detachment strategies were adopted (subjects should view the situation as fake or unreal and detach themselves from the situation). Contrary to some previous reports, we found that patients showed reduced activation, especially in the right-lateralized IFG, but not in the left-homologous region. However, this functional hemispheric asymmetry has also been found by other investigators reporting a greater role for the right hemisphere in the processing of negative affect in HCs (Davidson et al., 2002; Kim \& Hamann, 2007) and impairments in clinical conditions. Furthermore, Wager and colleagues (2008) showed that right vIPFC activity predicted drops in self-reported negative emotion, and that this relationship was independently mediated by separate pathways through the amygdala and the ventral striatum, thus stressing the importance of the right vIPFC for emotion regulation. Interestingly, neuroimaging studies report that activation in the vIPFC is strongly lateralized based on the type of stimuli processed (Levy and Wagner, 2011). Studies addressing the differences between upregulation and downregulation of negative emotions report that upregulation engaged primarily left-lateralized prefrontal regions, whereas downregulation engaged bilateral prefrontal regions (Kim \& Hamann, 2007).

In our study, we used strategies aimed at downregulating negative stimuli. Contrasting reappraisal versus view showed bilateral vIPFC activation in the control group. This is consistent with the pattern of rightlateralized activation for downregulation of negative emotions described by Ochsner et al. (2004). The altered right vIPFC activation in both patient groups implies an impaired cognitive control capacity of 
bottom-up systems, such as the amygdala that appraise the affective properties of stimuli. In general, the vIPFC - especially right vIPFC - is thought to play an important role in response inhibition as a particular form of executive control (Hampshire et al., 2010). Studies have highlighted the importance of the right hemisphere during cognitive reappraisal, engaging greater activation when the stimulus is more difficult and more cognitively demanding to reappraise (Ligeza et al., 2016). The correlation of right vIPFC activation with depressive and anxious symptomatology further supported the findings of the vIPFC as a key region involved in cognitive reappraisal across mood and anxiety disorders.

In addition to impairments across psychopathologies, (Zilverstand et al., 2016) reported deficits specific for mood and anxiety disorders. For patients with MDD, they found hyperactivity of the amygdala during downregulation of negative emotions, a finding in line with enhanced bottom-up responses and reduced top-down modulatory ability. Our ROI analysis indicated disorder-specific deficits for patients with MDD during cognitive reappraisal in the dmPFC, SMA, left middle temporal cortex and left precentral gyrus. These areas have been related to the execution of reappraisal, suggesting that a deficit in signaling the need for regulation in the vIPFC leads to less effective reappraisal (Kohn et al., 2014), a pattern specific to patients with MDD in our sample.

Regarding the neural correlates of emotion regulation deficits in PTSD, in addition to the reduced activation in the right IFG, the group of PTSD patients showed hypoactivation in the right dmPFC. Previous studies have focused on the structure, neurochemistry, and function of the amygdala, medial prefrontal cortex, and hippocampus in PTSD. Contrary to the hyperresponsiveness of the amygdala during emotion regulation, the medial prefrontal cortex appears to be volumetrically smaller and is hyporesponsive during symptomatic states and the performance of emotional cognitive tasks in PTSD (L. M. Shin et al., 2006). This finding is consistent with our results.

\section{Task-based and intrinsic functional connectivity}

FC analyses further investigated the importance of the group difference in the right IFG for cognitive reappraisal. In a task-based fMRI connectivity analysis using the right IFG as seed region, PTSD patients exhibited altered FC compared to HCs and MDD patients during reappraisal. Compared to HCs, PTSD patients showed increased FC of the rIFG with the left frontal orbital cortex, the left temporal pole, the left inferior frontal gyrus (pars opercularis), and the left frontal operculum. Interestingly, our results indicated altered connectivity predominantly in the PTSD group, suggesting higher FC in a fronto-temporo-parietal network. Higher connectivity in this network may result from a compensatory mechanism, taking into account that regions are important for emotion regulation and more specifically cognitive control mechanisms (Mathiak \& Weber, 2006). Although it may be speculative, this fronto-temporo-parietal hyperconnectivity may also reflect the use of suppression strategies that rely on engagement of this network. Furthermore, abnormalities on fronto-limbic connectivity have been the most discriminant feature for classification of PTSD based on the resting-state connectivity between the prefrontal and limbic regions (Liu et al., 2015). 
In addition, PTSD patients showed greater FC than MDD patients in regions including the precuneus, the anterior and posterior cingulate gyrus and the bilateral paracingulate gyrus. This finding is consistent with reports of PTSD patients showing increased FC in the anterior cingulate gyrus as a core component of the salience network (Chen et al., 2019). This may be related to the role played by the ACC in monitoring and appraisal of the external environment, reciprocally connecting brain regions to regulate stressor-related autonomic nervous system activity (Shin et al., 2013). The PCC is also implicated in stress neural circuit (Bremner, 2007), and researchers have found anatomical and functional change of this region in patients with PTSD (King et al., 2016; Zhang et al., 2012).

To investigate more general connectivity group differences, we employed an IC analysis as a whole-brain voxel-based connectivity measure that represents how well connected any given voxel is to the rest of the gray matter voxels in the brain. Compared to the conventional seed-based connectivity analyses, IC does not require a priori knowledge for the selection of the regions of interest (Martuzzi et al., 2011). In the present work, the comparison between HCs vs PTSD and PTSD vs MDD during cognitive reappraisal indicated IC abnormalities in PTSD patients. Previous studies have demonstrated impaired within- and between network FC in PTSD. For example, Akiki and colleagues (2017) report hypoactivity of the default mode network (DMN) and central executive network (CEN) that are putatively destabilized by an overactive and hyperconnected salience network (SN), which appears to be associated with an inefficient DMN-CEN modulation (Akiki et al., 2017). For the DMN network, we found that the group of PTSD patients differed from HCs in the activation of core regions such as the posterior cingulate cortex and precuneus. It has been suggested that DMN hypoactivity is associated with a deficit in the processing of autobiographical memory and self-references which may be a core underlying mechanism regarding PTSD symptomatology (Hayes et al., 2012).

\section{Limitations}

There are some limitations of the current study. First, the smaller sample size of PTSD compared to HCs and patients with MDD may have impeded the detection of significant differences between these groups. In addition, PTSD patients presented with relatively mild symptoms. And whereas most individuals in the MDD group were treated on the ward, a high percentage of PTSD patients was treated in an outpatient clinic. In addition, different subtypes of emotion regulation in PTSD may complicate interpretation of the data. A previous review described a model that includes these two types of emotion dysregulation in PTSD (Lanius et al., 2010). In this model, reexperiencing/hyperarousal reactivity is viewed as a form of emotion dysregulation that involves abnormally low activation in medial anterior brain regions, mediated by failure of prefrontal inhibition of limbic regions. In contrast, the dissociative subtype of PTSD is described as a form of emotion dysregulation that involves emotional overmodulation mediated by midline prefrontal inhibition of the same limbic regions. Both types of modulation are involved in a dynamic interplay and lead to alternating symptom profiles in PTSD (Lanius et al., 2010).

\section{Conclusions}


Presented results underscored the importance of the lateral PFC for cognitive reappraisal across mood and anxiety disorders. Patients with MDD and PTSD showed reduced activation within the right IFG. Supporting a key role of this region in psychopathology, reduced right IFG activity predicted a greater severity of anxiety or depression in both patients with MDD and PTSD. However, seed-based FC of the right IFG and IC patterns during cognitive reappraisal in PTSD patients were significantly different from those of HCs and MDD patients. In addition to hypoactivation of right IFG found in MDD and PTSD patients, these FC patterns suggested the underlying emotional processing of stimuli may show specific patterns for mood and anxiety disorders. Our findings underscore the importance of the lateral PFC for cognitive reappraisal and further suggest that the right IFG may be a suitable target for fMRI-based neurofeedback or other neuromodulatory interventions that may contribute to an improvement of symptoms.

\section{Declarations}

\section{Acknowledgments}

This study was supported by the German Ministry for Education and Research [BMBF; APIC: 01EE1405A, B, and C; TRAM: 01DN18026t]; German Research Foundation [DFG; IRTG 2150, MA 2631/6-1]; and the Interdisciplinary Center for Clinical Research (IZKF) of the University Hospital Aachen. We thank Sarah Bleich, Arndt Brandl and Manouela Kosmadaki for their assistance with participant recruitment and data collection. The authors report no further financial disclosures or potential conflicts of interest.

\section{Authors' contributions}

MK, JZ, MZ, KM contributed to conception and design of the study. MK, RM and JI were involved in the investigation. MK, RM and JZ performed the statistical analysis. KM supervised the measurements and statistical data analysis. All authors contributed to the writing of the manuscript, revised, read, and approved the submitted version.

\section{Funding}

This study was supported by the German Ministry for Education and Research [BMBF; APIC: 692 01EE1405A, B, and C; TRAM: 01DN18026t] and the German Research Foundation [DFG; IRTG 2150, MA 2631/6-1].

\section{Availability of data and materials}

The data that support the findings of this study are available from RWTH Aachen University Hospital, but restrictions apply to the availability of these data, which were used under license for the current study, and so are not publicly available. Data are however available from the authors upon reasonable request and with permission of RWTH Aachen University Hospital.

\section{Ethics approval and consent to participate}


All experiments were performed with the written informed consent of each participant and approval by the local Ethics Committee of the RWTH Aachen University Hospital, in line with the Code of Ethics of the World Medical Association (Declaration of Helsinki).

\section{Consent for publication}

Not applicable.

\section{Competing interests}

The authors declare that they have no competing interests.

\section{References}

1. Akiki, T. J., Averill, C. L., \& Abdallah, C. G. (2017). A network-based neurobiological model of PTSD: evidence from structural and functional neuroimaging studies. Current Psychiatry Reports, 19(11), $1-10$.

2. Association American Psychiatric. (2000). Diagnostic and statistical manual of mental disorders (4th ed., t). https://doi.org/doi:10.1176/appi.books.9780890423349

3. Berking, M., Wirtz, C. M., Svaldi, J., \& Hofmann, S. G. (2014). Successful Emotion Regulation Skills Application Negatively Predicts Depressive Symptom Severity over Five Years in Individuals Reporting at least some Depressive Symptoms at Baseline. Behaviour Research and Therapy. https://doi.org/10.1016/j.brat.2014.03.003

4. Bremner, J. D. (2007). Functional neuroimaging in post-traumatic stress disorder. Expert Review of Neurotherapeutics, 7(4), 393-405.

5. Buhle, J. T., Silvers, J. A., Wager, T. D., Lopez, R., Onyemekwu, C., Kober, H., Weber, J., \& Ochsner, K. N. (2014). Cognitive Reappraisal of Emotion: A Meta-Analysis of Human Neuroimaging Studies. Cerebral Cortex, 24, 2981-2990. https://doi.org/10.1093/cercor/bht154

6. Chen, H. J., Zhang, L., Ke, J., Qi, R., Xu, Q., Zhong, Y., Pan, M., Li, J., Lu, G. M., \& Chen, F. (2019). Altered resting-state dorsal anterior cingulate cortex functional connectivity in patients with post-traumatic stress disorder. Australian \& New Zealand Journal of Psychiatry, 53(1), 68-79.

7. Costafreda, S. G., Brammer, M. J., David, A. S., \& Fu, C. H. (2008). Predictors of amygdala activation during the processing of emotional stimuli: A meta-analysis of 385 PET and fMRI studies. Brain Res Brain Res Rev, 58, 57-70.

8. Cristea, I. A., Kok, R. N., \& Cuijpers, P. (2015). Efficacy of cognitive bias modification interventions in anxiety and depression: meta-analysis. The British Journal of Psychiatry, 206(1), 7-16.

9. Davidson, R. J., Lewis, D. A., Alloy, L. B., Amaral, D. G., Bush, G., Cohen, J. D., Drevets, W. C., Farah, M. J., Kagan, J., Mcclelland, J. L., Nolen-hoeksema, S., \& Peterson, B. S. (2002). Neural and Behavioral Substrates of Mood and Mood Regulation. Biological Psychiatry, 52(6), 478-502. 
10. Dickie, E. W., Brunet, A., Akerib, V., \& Armony, J. L. (2013). Anterior cingulate cortical thickness is a stable predictor of recovery from post-traumatic stress disorder. Psychological Medicine, 43(3), 645653.

11. Etkin, A., \& Wager, T. D. (2007). Functional neuroimaging of anxiety: A metaanalysis of emotional processing in PTSD, social anxiety disorder, and specific phobia. Am J Psychiatry, 164, Am J Psychiatry.

12. Fischer, A. S., Keller, C. J., \& Etkin, A. (2016). The clinical applicability of functional connectivity in depression: Pathways toward more targeted intervention. Biological Psychiatry: Cognitive Neuroscience and Neuroimaging, 1(3), 262-270.

13. Gross, J. J., \& John, O. P. (2003). Individual differences in two emotion regulation processes: Implications for affect, relationships, and well-being. J Pers Soc Psychol, 85, 348-362.

14. Hampshire, A., Chamberlain, S. R., Monti, M. M., Duncan, J., \& Owen, A. (2010). The role of the right inferior frontal gyrus: Inhibition and attentional control. Neuroimage, 50(3), 1313-1319. https://doi.org/10.1016/j.neuroimage.2009.12.109

15. Hayes, J. P., Hayes, S. M., \& Mikedis, A. M. (2012). Quantitative meta-analysis of neural activity in posttraumatic stress disorder. Biology of Mood \& Anxiety Disorders, 2(1), 1-13.

16. Hofmann, S. G. (2014). Interpersonal Emotion Regulation Model of Mood and Anxiety Disorders. Cogn Ther Res. https://doi.org/10.1007/s10608-014-9620-1

17. Insel, T., Cuthbert, B., Garvey, M., Heinssen, R., Pine, D., Quinn, K., Sanislow, C., \& Wang, P. (2010). Research Domain Criteria (RDoC): toward a new classification framework for research on mental disorders. American Journal of Psychiatry, 167(7), 748-751. https://doi.org/doi.org/10.1176/appi.ajp.2010.09091379.

18. Kaiser, R. H., Andrews-Hanna, J. R., Wager, T. D., \& Pizzagalli, D. A. (2015). Large-scale network dysfunction in major depressive disorder: a meta-analysis of resting-state functional connectivity. JAMA Psychiatry, 72(6), 603-611.

19. Kanske, P., Heissler, J., Schönfelder, S., \& Wessa, M. (2012). Neural correlates ofemotion regulation deficits in remitted depression: The influence of regulation strategy, habitual regulation use, and emotional valence. Neurolmage, 61(3), 686-693. https://doi.org/10.1016/j.neuroimage.2012.03.089

20. Keller, M., Zweerings, J., Klasen, M., Zvyagintsev, M., Iglesias-Fuster, J., Mendoza Quiñones, R., \& Mathiak, K. (2021). fMRI neurofeedback-enhanced cognitive reappraisal training in depression: a double-blind comparison of left and right vIPFC regulation. Frontiers in Psychiatry, 1457. https://doi.org/10.3389/fpsyt.2021.715898

21. Kim, S. H., \& Hamann, S. (2007). Neural correlates of positive and negative emotion regulation. Journal of Cognitive Neuroscience, 19(5), 776-798.

22. King, A. P., Block, S. R., Sripada, R. K., Rauch, S., Giardino, N., Favorite, T., Angstadt, M., Kessler, D., Welsh, R., \& Liberzon, I. (2016). Altered default mode network (DMN) resting state functional connectivity following a mindfulness-based exposure therapy for posttraumatic stress disorder (PTSD) in combat veterans of Afghanistan and Iraq. Depression and Anxiety, 33(4), 289-299. 
23. Kohn, N., Eickhoff, S. B., Scheller, M., Laird, A. R., Fox, P. T., Habel, U., Medicine, T. B., Psychology, M., Antonio, S., \& Antonio, S. (2014). Neural network of cognitive emotion regulation - An ALE metaanalysis and MACM analysis. Neuroimage, 15(87), 345-355.

https://doi.org/10.1016/j.neuroimage.2013.11.001.Neural

24. Lang, P. J., Bradley, M. M., \& Cuthbert, B. N. (2008). International affective picture system (IAPS): affective ratings of pictures and instruction manual. University of Florida, Gainesville. Tech Rep A-8.

25. Lanius, R. A., Vermetten, E., Loewenstein, R. J., Brand, B., Schmahl, C., Bremner, D., \& Spiegel, D. (2010). Emotion Modulation in PTSD: Clinical and Neurobiological Evidence for a Dissociative Subtype. Am J Psychiatry, 167(6), 640-647.

https://doi.org/10.1176/appi.ajp.2009.09081168.Emotion

26. Levy, B. J., \& Wagner, A. D. (2011). Cognitive control and right ventrolateral prefrontal cortex : reflexive reorienting, motor inhibition, and action updating. ANNALS OF THE NEW YORK ACADEMY OF SCIENCES, 1224, 40-62. https://doi.org/10.1111/j.1749-6632.2011.05958.x

27. Ligeza, T. S., Wyczesany, M., Tymorek, A. D., \& Kamiński, M. (2016). Interactions between the prefrontal cortex and attentional systems during volitional affective regulation: an effective connectivity reappraisal study. Brain Topography, 29(2), 253-261.

https://doi.org/doi.org/10.1007/s10548-015-0454-2

28. Liu, F., Xie, B., Wang, Y., Guo, W., Fouche, J.-P., Long, Z., Wang, W., Chen, H., Li, M., \& Duan, X. (2015). Characterization of post-traumatic stress disorder using resting-state fMRI with a multi-level parametric classification approach. Brain Topography, 28(2), 221-237.

29. Marques, L. M., Morello, L. Y. N., \& Boggio, P. S. (2018). Ventrolateral but not dorsolateral prefrontal cortex tDCS effectively impact emotion reappraisal-effects on emotional experience and interbeat interval. Scientific Reports, 8(1), 1-12.

30. Martuzzi, R., Ramani, R., Qiu, M., Shen, X., Papademetris, X., \& Constable, R. T. (2011). A whole-brain voxel based measure of intrinsic connectivity contrast reveals local changes in tissue connectivity with anesthetic without a priori assumptions on thresholds or regions of interest. Neuroimage, 58(4), 1044-1050.

31. Mathiak, K., \& Keller, M. (2021). Clinical Application of Real-Time fMRI-Based Neurofeedback for Depression. Advances in Experimental Medicine and Biology, 1305, 275-293. https://doi.org/10.1007/978-981-33-6044-0_15

32. Mathiak, K., \& Weber, R. (2006). Toward brain correlates of natural behavior: fMRI during violent video games. Human Brain Mapping, 27(12), 948-956.

33. Nusslock, R., Walden, K., \& Harmon-jones, E. (2015). Asymmetrical frontal cortical activity associated with differential risk for mood and anxiety disorder symptoms: An RDoC perspective. International Journal of Psychophysiology, 98(2), 249-261. https://doi.org/10.1016/j.ijpsycho.2015.06.004

34. Ochsner, K N, \& Gross, J. J. (2005). The cognitive control of emotion. Trends Cogn. Sci., 9, $242-249$.

35. Ochsner, Kevin N, Ray, R. D., Cooper, J. C., Robertson, E. R., Chopra, S., Gabrieli, J. D. E., \& Gross, J. J. (2004). For better or for worse: neural systems supporting the cognitive down- and up-regulation of 
negative emotion. Neurolmage, 23, 483-499. https://doi.org/10.1016/j.neuroimage.2004.06.030

36. Phillips, M., Ladouceur, C., \& Drevets, W. (2008). A neural model of voluntary and automatic emotion regulation: implications for understanding the pathophysiology and neurodevelopment of bipolar disorder. Mol. Psychiatry, 13, 829-857. https://doi.org/doi:10.1038/mp.2008.65.A

37. Picó-Pérez, M., Radua, J., Steward, T., Menchón, J. M., \& Soriano-Mas, C. (2017). Emotion regulation in mood and anxiety disorders: A meta-analysis of fMRI cognitive reappraisal studies. Progress in Neuro-Psychopharmacology and Biological Psychiatry, 79, 96-104.

https://doi.org/10.1016/j.pnpbp.2017.06.001

38. Price, J. L., \& Drevets, W. C. (2010). Neurocircuitry of mood disorders. Neuropsychopharmacology, 35(1), 192-216.

39. Rabinak, C. A., MacNamara, A., Kennedy, A. E., Angstadt, M., Stein, M. B., Liberzon, I., \& Phan, K. L. (2014). Focal and Aberrant Prefrontal Engagement During Emotion Regulation in Veterans With Posttraumatic Stress Disorder. Depression and Anxiety, 861(February), 851-861.

https://doi.org/10.1002/da.22243

40. Raichle, M. E. (2015). The brain's default mode network. Annual Review of Neuroscience, 38, 433447. https://doi.org/10.1146/annurev-neuro-071013-014030

41. Rive, M. M., van Rooijen, G., Veltman, D. J., Phillips, M. L., Schene, A. H., \& Ruhé, H. G. (2013). Neural correlates of dysfunctional emotion regulation in major depressive disorder. A systematic review of neuroimaging studies. Neurosci. Biobehav. Rev., 37, 2529-2553.

https://doi.org/doi:10.1016/j.neubiorev.2013.07.018

42. Rytwinski, N. K., Scur, M. D., Feeny, N. C., \& Youngstrom, E. A. (2013). The co-occurrence of major depressive disorder among individuals with posttraumatic stress disorder: A meta-analysis. Journal of Traumatic Stress, 26(3), 299-309.

43. Sarkheil, P., Zilverstand, A., Kilian-Hütten, N., Schneider, F., Goebel, R., \& Mathiak, K. (2015). fMRI feedback enhances emotion regulation as evidenced by a reduced amygdala response. Behavioural Brain Research, 281, 326-332.

44. Shin, L. M., Rauch, S. L., \& Pitman, R. K. (2006). Amygdala, medial prefrontal cortex, and hippocampal function in PTSD. Annals of the New York Academy of Sciences, 1071(1), 67-79.

45. Shin, Y.-W., Dzemidzic, M., Jo, H. J., Long, Z., Medlock, C., Dydak, U., \& Goddard, A. W. (2013). Increased resting-state functional connectivity between the anterior cingulate cortex and the precuneus in panic disorder:: Resting-state connectivity in panic disorder. Journal of Affective Disorders, 150(3), 1091-1095.

46. Sripada, R. K., King, A. P., Garfinkel, S. N., Wang, X., Sripada, C. S., Welsh, R. C., \& Liberzon, I. (2012). Altered resting-state amygdala functional connectivity in men with posttraumatic stress disorder. Journal of Psychiatry \& Neuroscience: JPN, 37(4), 241. https://doi.org/10.1503/jpn.110069

47. Stanley, I. H., Hom, M. A., Spencer-Thomas, S., \& Joiner, T. E. (2017). Examining anxiety sensitivity as a mediator of the association between PTSD symptoms and suicide risk among women firefighters. Journal of Anxiety Disorders, 50, 94-102. https://doi.org/doi.org/doi:10.1016/j.janxdis.2017.06.003 
48. Statsoft, I. N. C. (2011). STATISTICA (data analysis software system), version 10.0. StatSoft Tulsa^ eOklahoma Oklahoma.

49. Wager, T. D., Davidson, M. L., Hughes, B. L., Lindquist, M. A., \& Ochsner, K. N. (2008). PrefrontalSubcortical Pathways Mediating Successful Emotion Regulation. Neuron, 59, 1037-1050. https://doi.org/10.1016/j.neuron.2008.09.006

50. Wang, X., Feng, Z., Zhou, D., Lei, X., Liao, T., Zhang, L., Ji, B., \& Li, J. (2014). Dissociable Self Effects for Emotion Regulation: A Study of Chinese Major Depressive Outpatients. BioMed Research International, 2014, 1-11. https://doi.org/dx.doi.org/10.1155/2014/390865

51. Weiskopf, N., Scharnowski, F., Veit, R., Goebel, R., Birbaumer, N., \& Mathiak, K. (2004). Self-regulation of local brain activity using real-time functional magnetic resonance imaging (fMRI). Journal of Physiology-Paris, 98(4-6), 357-373.

52. Zhang, L., Li, W., Shu, N., Zheng, H., Zhang, Z., Zhang, Y., He, Z., Hou, C., Li, Z., \& Liu, J. (2012). Increased white matter integrity of posterior cingulate gyrus in the evolution of post-traumatic stress disorder. Acta Neuropsychiatrica, 24(1), 34-42.

53. Zigmond, A. S., \& Snaith, R. P. (1983). The hospital anxiety and depression scale. Acta Psychiatrica Scandinavica, 67(6), 361-370.

54. Zilverstand, A., Parvaz, M. A., \& Goldstein, R. Z. (2016). Neuroimaging cognitive reappraisal in clinical populations to define neural targets for enhancing emotion regulation. A systematic review. Neurolmage. https://doi.org/10.1016/j.neuroimage.2016.06.009

55. Zilverstand, A., Parvaz, M. A., \& Goldstein, R. Z. (2017a). Neuroimaging cognitive reappraisal in clinical populations to define neural targets for enhancing emotion regulation. A systematic review. Neuroimage, 151, 105-116.

56. Zilverstand, A., Parvaz, M. A., \& Goldstein, R. Z. (2017b). Neuroimaging cognitive reappraisal in clinical populations to define neural targets for enhancing emotion regulation. A systematic review. Neurolmage, 151, 105-116. https://doi.org/10.1016/j.neuroimage.2016.06.009

57. Zweerings, J., Mathiak, K., Zvyagintsev, M., Turetsky, B. I., Klasen, M., König, A. A., Roecher, E., \& Gaebler, A. J. (2019). Fronto-parietal and temporal brain dysfunction in depression: A fMRI investigation of auditory mismatch processing. Neuroimage, 151(October 2018), 3657-3668. https://doi.org/10.1002/hbm.24623

58. Zweerings, J., Pflieger, E. M., Mathiak, K. A., Zvyagintsev, M., Kacela, A., Flatten, G., \& Mathiak, K. (2018). Impaired voluntary control in PTSD: probing self-regulation of the ACC with real-time fMRI. Frontiers in Psychiatry, 9, 219.

59. Zweerings, J., Sarkheil, P., Keller, M., Dyck, M., Klasen, M., Becker, B., Gaebler, A. J., Ibrahim, C. N., Turetsky, B. I., \& Zvyagintsev, M. (2020). Rt-fMRI neurofeedback-guided cognitive reappraisal training modulates amygdala responsivity in posttraumatic stress disorder. Neurolmage: Clinical, 28, 102483.

\section{Figures}




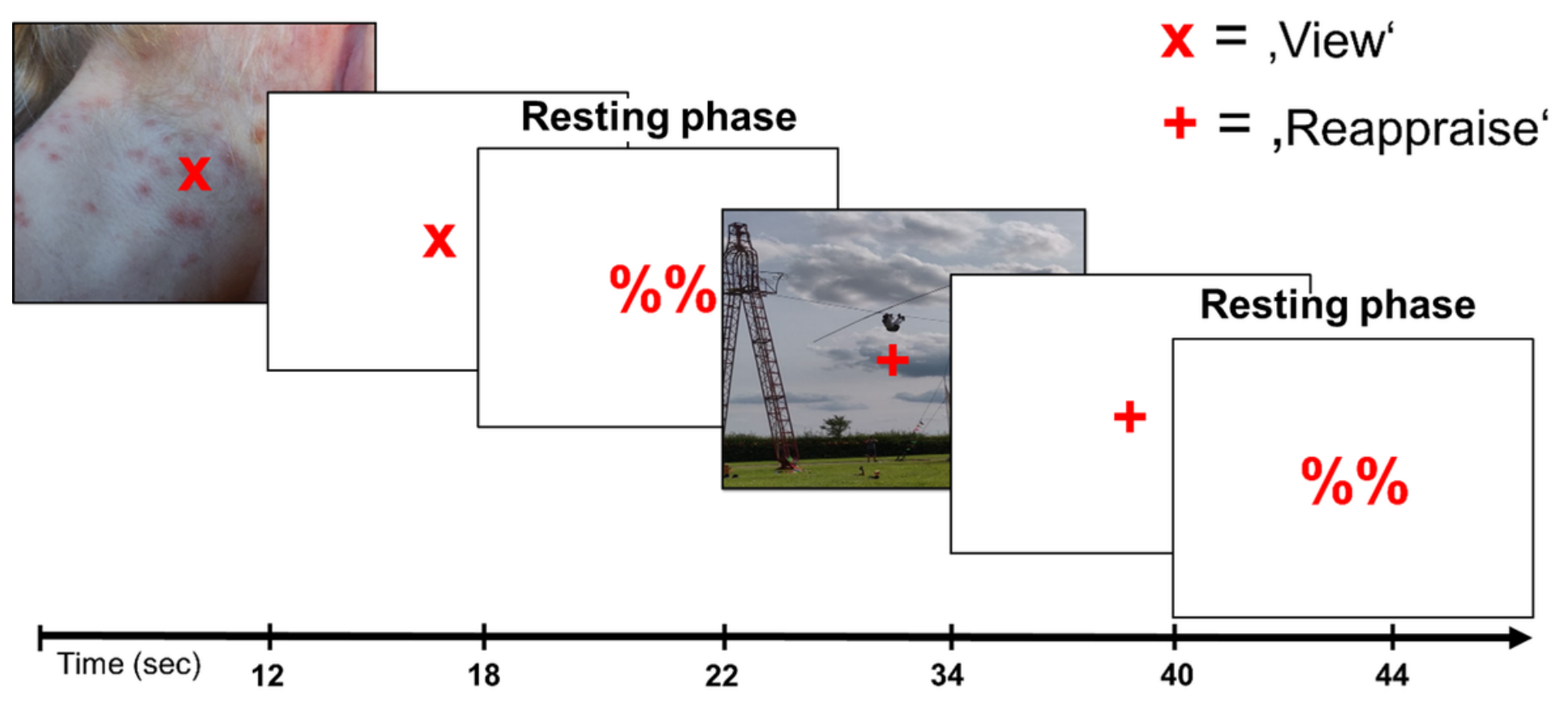

Figure 1

Experimental design. Participants completed 9 reappraise-view cycles with intermittent resting phases. The task was to either maintain the negative emotion elicited by a picture ('view' condition) or to change negative emotions of pictures by using cognitive reappraisal strategies such as imagining that (1) the situation is not as bad as it looks or (2) will get better in the future or by (3) imagining that the situation is not real or (4) taking the perspective of a professional.

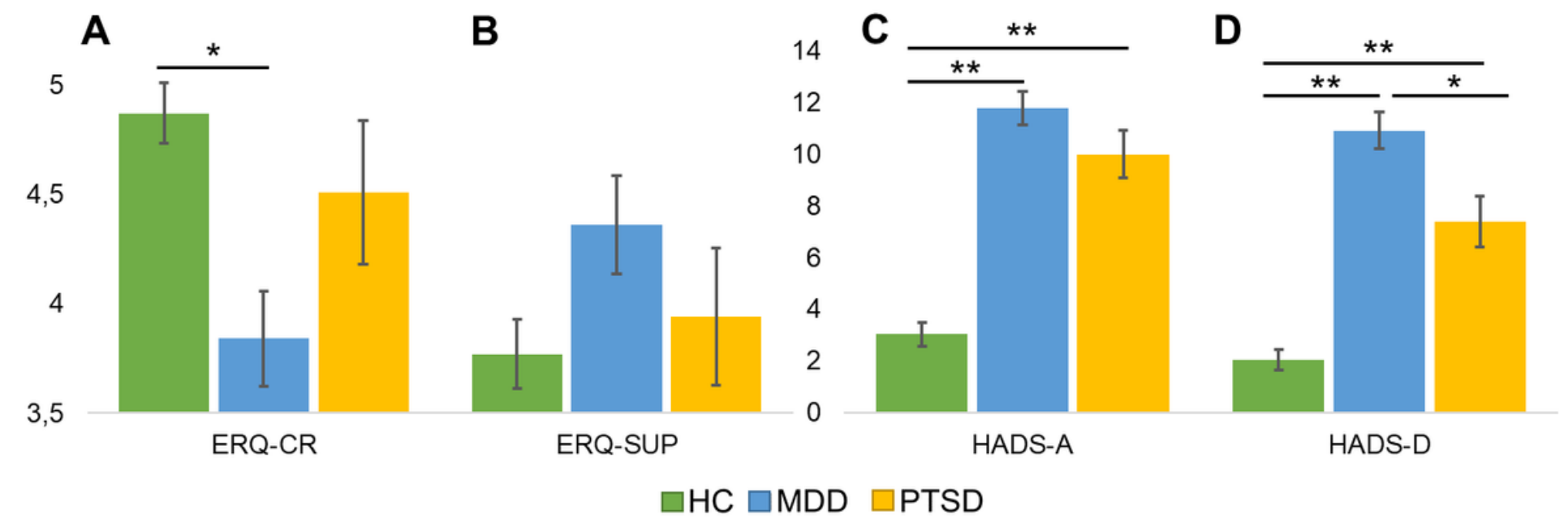

\section{Figure 2}

Mean rating scores of clinical scales in healthy individuals, patients with depression and PTSD. A) Patients with depression used significantly less cognitive reappraisal than healthy individuals. B) The three groups showed a similar tendency to use suppression strategies. C) MDD and PSTD patients showed higher levels of anxiety compared to healthy controls. D) MDD patients had the highest scores of depressive symptoms. Error bars denote standard errors of the mean. Significance: * $=p<.01$; $* *=p<$ 
.001. Abbreviations: ERQ: Emotion regulation questionnaire (CR: cognitive reappraisal, SUP: suppression); HADS: Hospital anxiety and depression scale (A: anxiety, D: depression); HC: Healthy controls; MDD: Major depressive disorder; PTSD: Post-traumatic stress disorder.

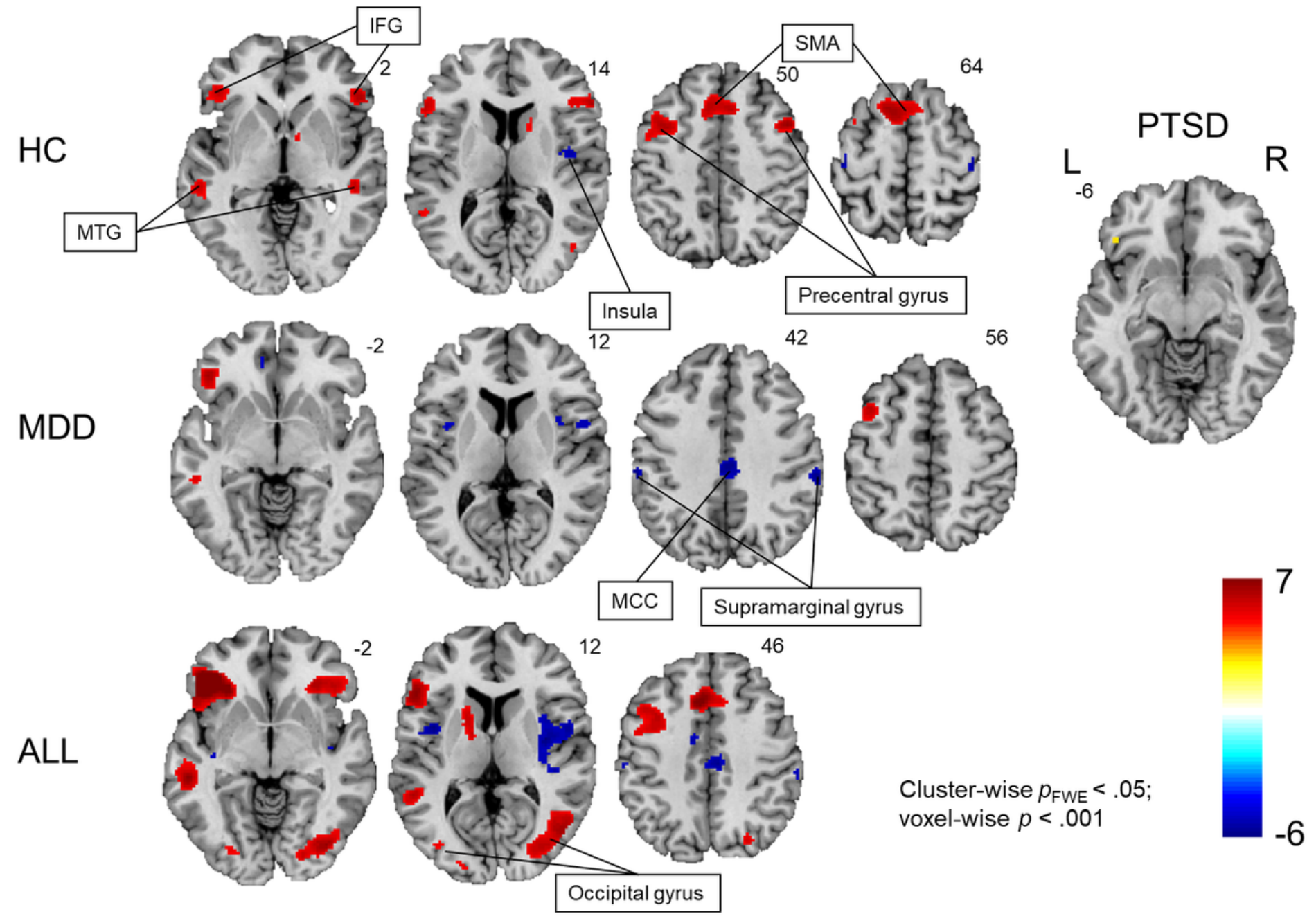

\section{Figure 3}

Whole-brain functional brain maps depicting increased activation (reappraise $>$ view) and decreased activation (view > reappraise) during cognitive reappraisal for healthy controls (HCs), patients with major depressive disorder (MDD) and patients with post-traumatic stress disorder (PTSD). Activation in the left IFG was found in all groups while activation during cognitive reappraisal was most extensive in HCs.
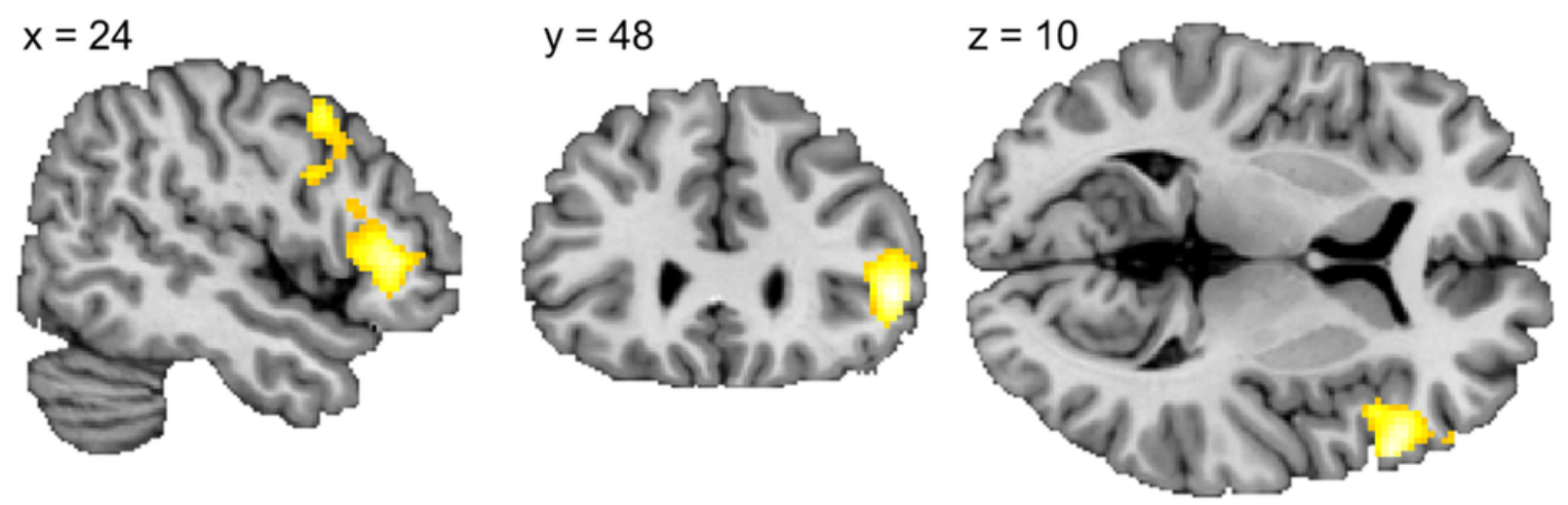


\section{Figure 4}

Higher brain activation in the right triangular and opercular parts of the IFG in healthy individuals compared to patients (MDD and PTSD) for the contrast reappraise $>$ view (TPeak $=4.96$, extend threshold $=783$ voxels). Results are corrected at $\mathrm{p}<.001$ (unc.) with cluster-correction at $\mathrm{pFWE}<.05$.
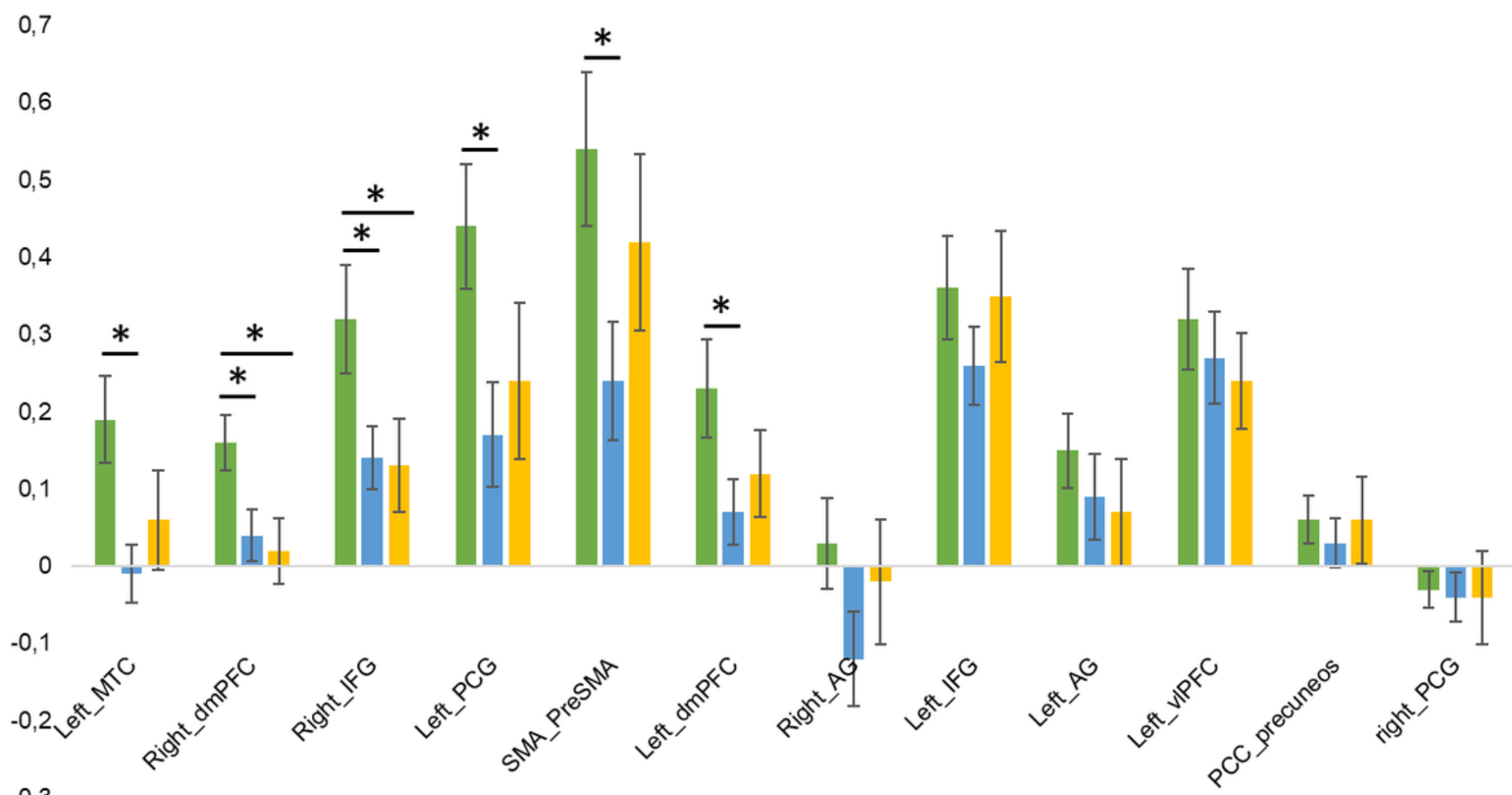

$$
=\mathrm{HC} \equiv \mathrm{MDD}=\mathrm{PTSD}
$$

\section{Figure 5}

Comparison of ROI peak activity between groups (healthy controls (HCs), major depressive disorder (MDD) and post-traumatic stress disorder (PTSD)). Beta values were extracted for the contrast 'reappraise > view' for each region of interest. Significantly higher beta values (Tukey's correction) for HCs compared to MDD patients were found in the left middle temporal cortex, bilateral dorsomedial PFC, right inferior frontal gyrus, supplementary motor cortex. Furthermore, beta values were significantly higher for HCs compared to PTSD patients in the right dorsomedial PFC and right IFG. Error bars display standard errors of mean. 
HCs
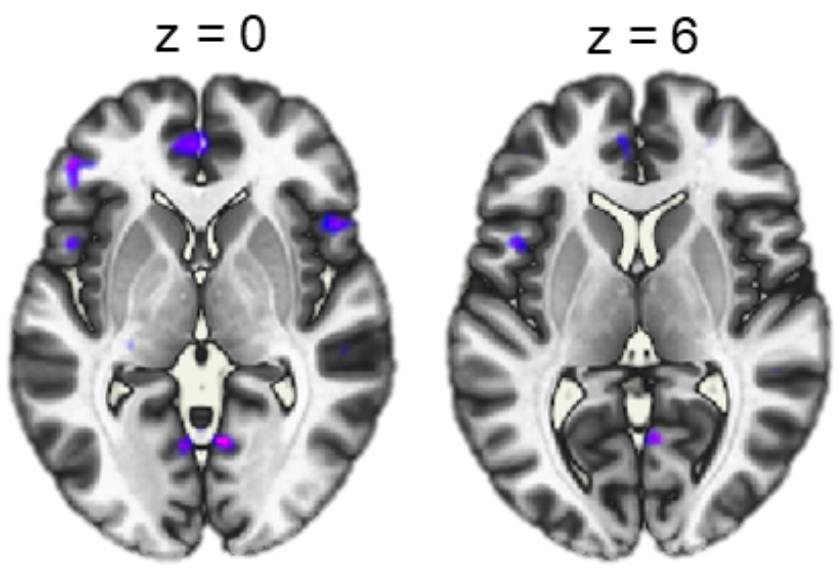

$z=18$
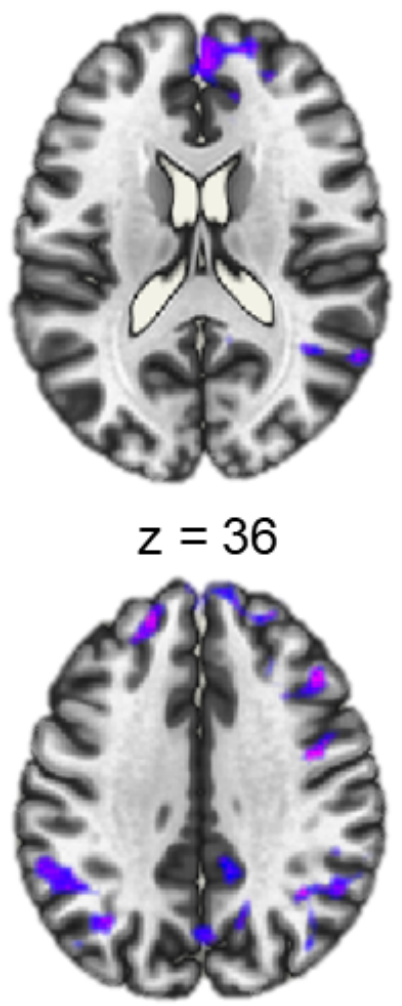
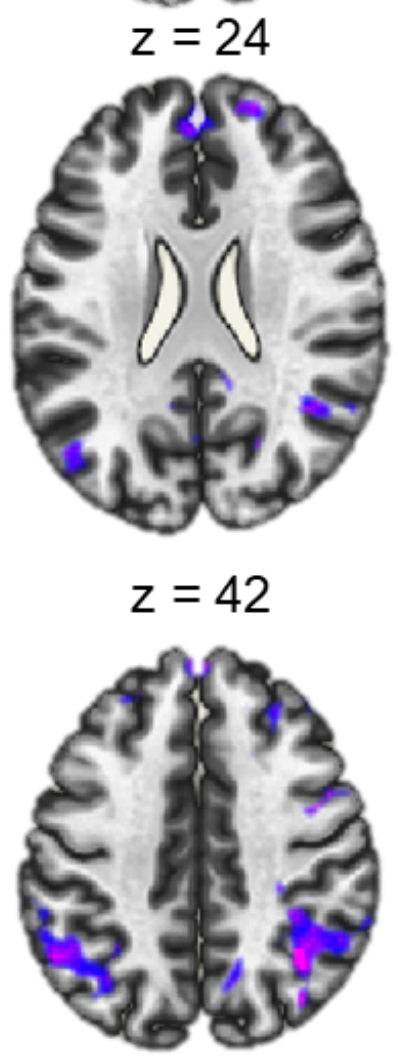

MDD
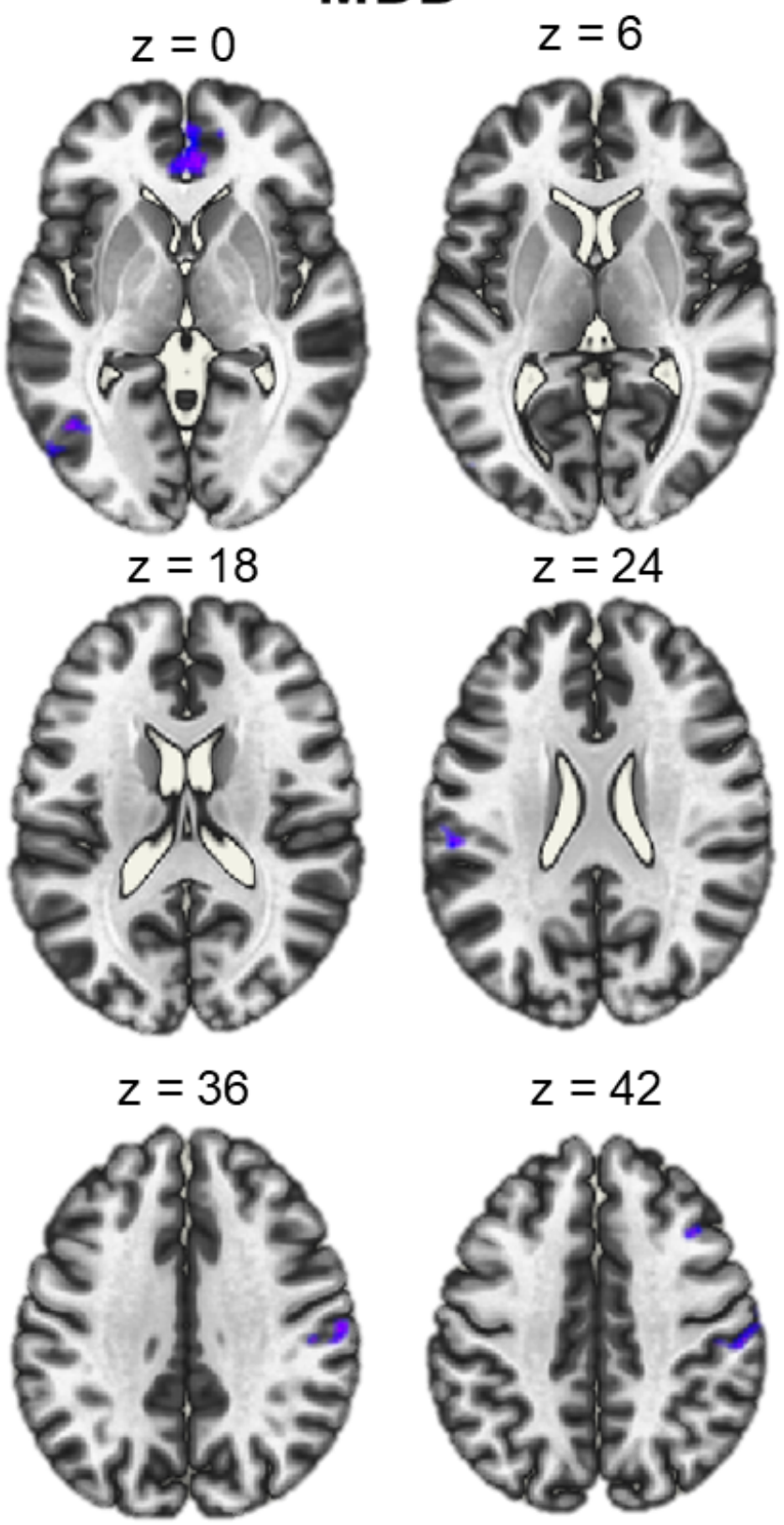

Figure 6

Intrinsic connectivity (IC) in healthy controls (HCs) and patients with major depressive disorder (MDD). The whole-brain voxel-based connectivity revealed reduced connectivity of neural networks during the cognitive reappraisal task (contrast between 'reappraise vs. view'). The significant threshold was $p<.005$ uncorrected voxel level and with $\mathrm{p}<.05 \mathrm{FWE}$-correction at the cluster level. HCs showed a wide-spread neural network distribution of IC during the task. In contrast, patients with MDD showed less pattern of activation during the task. No clusters in the post-traumatic stress disorder group survived correction.

\section{Supplementary Files}


This is a list of supplementary files associated with this preprint. Click to download.

- Supplmaterial.docx 